\title{
EL NUEVO ESPACIO SOCIAL DE TARAPACÁ: LAS CLAVES DE UNA NUEVA SOCIABILIDAD
}

\author{
THE NEW SOCIAL SPACE TARAPACÁ: THE KEYS TO A NEW SOCIABILITY
}

\author{
Víctor Guerrero Cossio*
}

\begin{abstract}
Este artículo trata de la transformación del espacio social en la región de Tarapacá, especialmente en las comunidades adyacentes a los yacimientos mineros y en la ciudad de Iquique, debido al impacto territorial sufrido como consecuencia de la ejecución de los proyectos mineros activados desde 1995 .

La modernización de los procesos tecnológicos y el crecimiento económico son factores virtuosos de este proceso, sin embargo la desigual distribución de los beneficios, expresada en salarios, viviendas, transporte, educación y equipamiento tecnológico, revela un aspecto vicioso que produce un espacio social segmentado y excluyente.
\end{abstract}

Palabras claves: Espacio social, territorio, minería, impacto sociocultural rural y urbano.

This article deals with the transformation of the social space in the region of Tarapacá, particularly within the communities close to mines, as well as in the city of Iquique, due to the territorial impact of the implementation of mining projects from 1995.

The modernization of technological processes and economic growth are the positive factors in the development of these processes. However, the unequal distribution of their benefits, namely in salaries, housing, transport, education and technological equipment reveals some vicious aspects that produce a segmented and excluding social space.

Key words: Social space, territory, mining, rural and urban socio-cultural impact.

\section{Introducción}

\section{Antecedentes Económicos y Sociales}

Desde la década del 80 se inicia en la región de Tarapacá un nuevo ciclo minero, en este caso principalmente de los proyectos mineros cupríferos y caracterizados por la trasnacionalización de esta actividad en Chile. Estos se encuentran en ejecución desde los años 90, operan en la provincia del Tamarugal y se han transformado en un importante factor de cambios, tanto en los aspectos materiales como en los inmateriales.

Desde un punto de vista crítico, los beneficios económicos del capital minero sobre la realidad regional son fugaces, concentrados y excluyentes, debido a la autonomía que tiene esta actividad y la débil regulación legal y redistribución económica estatal. Además la lógica de este capital es desencadenante de efectos adversos profundos y de larga duración, como son la potenciación de migraciones desde las comunidades tradicionales, la externalización de costos residenciales con cargo al Estado, creación de una aristocracia laboral en términos de consumo económico-social y producción de un colectivo social frágil y desarraigado de los intereses regionales. Estas condiciones determinan la creación del espacio social en las colectividades humanas relacionadas directamente con la circulación del capital minero transnacional.

El impacto sociocultural del capital minero tiene notorios efectos sobre el conjunto de la realidad regional, pero especialmente sobre los asentamientos humanos más relacionados con su actividad. Se trata de localidades rurales y urbanas vinculadas a las operaciones mineras, sea por su cercanía geográfica o por la relación económica que establece su población en diversos momentos con dichos proyectos - exploración, montaje, producción- productivos. Así, la dinámica producida por el capital minero altera las relaciones sociales tradicionales existentes en los poblados de Huatacondo y Colonia Pintados, como también diferencia la relación con la ciudad de los trabajadores mineros que residen en los condominios de Iquique respecto de los otros residentes de la localidad. En el primer caso se pierde la relación comunitaria y en el segundo produce una escisión con los intereses de la ciudad.

Se sostiene que en la actualidad el fenómeno de la globalización cambia las coordenadas de la

* Universidad Arturo Prat, Facultad de Ciencias Humanas, Iquique, Chile. Correo electrónico: victor.guerrero@unap.cl 
sociabilidad de una manera relativamente homogénea, pero en el caso de las poblaciones mencionadas se agrega a dicho influjo la relación específica producida por el vínculo con los proyectos mineros y su diseño organizacional.

El eje conceptual de la sociabilidad permite una perspectiva espacial, topológica, de las relaciones sociales existentes en el medio regional. Permite analizar la crisis social expresada espacialmente en tanto transformación profunda del orden imperante, del sistema social y del mundo de vida.

En este sentido, los aspectos más relevantes a considerar en la investigación del espacio social transformado son los siguientes:

- Gran movilidad poblacional y aumento del peso extrarregional en su composición, afectando las bases tradicionales de la interacción social, la identidad regional y generando vínculos nuevos que se destacan por ser efímeros y superficiales. Esto incide en nuevas dinámicas de asimilación de la población andina, a causa de los nuevos y cada vez más sólidos lazos con el mundo urbano y moderno.

- Modernización de los sistemas de transportes y comunicaciones, que se traducen en menor arraigo con la región y con las localidades, como asimismo mayor vinculación con el mundo exterior, ya que la accesibilidad es mayor y las redes de interacción son más extensas.

- Recomposición amplia de la población rural de las zonas altas de la región, por el aumento móvil o permanente de agentes mineros y de la burocracia estatal. La relación urbana-rural se transforma por la presencia de poblaciones exógenas y por los mejores medios que tienen las comunidades aymaras para vincular sus actividades entre la ciudad y la comunidad originaria.

- Cambio del carácter tradicional del campamento minero, también condicionado por las transformaciones profundas que permite el nivel tecnológico alcanzado por la sociedad moderna.

- De un asentamiento humano íntimamente ligado a la faena, donde el obrero residía en él junto a su familia, se pasa a una diferenciación nítida de la residencia laboral y familiar, donde ahora el trabajador permanece la mitad de su tiempo en el campamento laboral y la otra mitad en el campamento familiar, que es de tipo condominio.
- Transformación de las relaciones espaciales, caracterizándose ahora como dinámicas de flujo más que de lugares. En este sentido las relaciones entre los distintos asentamientos humanos, sean laborales, residenciales, urbanos y rurales, ya no son distantes ni distintos, porque las modernas tecnologías de transportes y comunicaciones potencian la idea de homogeneidad en cuanto a las demandas de consumo económico y social.

- Paradójicamente, las localidades que tienen como centro exclusivo y hegemónico a la ciudad, pero con altas posibilidades de vinculación con ella, tienden a la heterogeneidad por sobre la homogeneidad social, produciéndose a la vez la pérdida de autonomía a causa de la heteronomía resultante de la diversidad y al hecho de que el capital principal es externo y escasamente controlado.

Debido a esa relación contradictoria entre la tendencia a cierta homogeneidad de consumo, con una heteronomía respecto del producto, en un marco de creciente heterogeneidad poblacional, se refuerza el carácter de ciudad bajo la lógica de espacio-flujo (dinámico) más que de espacio-lugar (estático). Se quiebra definitivamente el sentido de comunidad y aparece una nueva sociabilidad, definida por los fenómenos de diversidad, segmentación y exclusión, tanto en la población aledaña a las operaciones mineras como aquella residente en la ciudad.

En este sentido indicadores como el ingreso, consumo y condiciones de habitabilidad se relacionarán con el tipo de vínculos entre los residentes y de estos con el medio local, reconociendo así el carácter de la sociabilidad, la cohesión social y la identidad cultural, predominante en los espacios sociales referidos.

\section{Antecedentes poblacionales de la región de Tarapacá}

La región de Tarapacá limita al oeste con el océano Pacífico, al este con la república de Bolivia, al norte con la república de Perú y al sur con la región de Antofagasta. La región de Tarapacá tiene una superficie de $42.225,8 \mathrm{~km}^{2}$, con una población de 238.950 habitantes. Su densidad poblacional es de 5,65 habitantes $/ \mathrm{km}^{2}$. La región cuenta administrativamente con las provincias de Tamarugal e Iquique, en cuyo interior se alojan siete comunas. 
La capital regional es Iquique y cuenta con 300.021 habitantes, concentrando al 90,6\% de la población total de Tarapacá. Las siguientes ciudades más pobladas son Pozo Almonte y Pica, con 10.830 y 6.178 habitantes, respectivamente (Censo 2012).

La región de Tarapacá tiene un indudable perfil urbano, pero también muestra crecimiento de dos localidades rurales, Pozo Almonte y Pica, Sin embargo, este incremento obedece a un patrón no relacionado con la revitalización de la población aymara, sino a efectos de la minería. Las restantes comunas rurales (3) no asociadas al capital minero y las localidades adyacentes a los yacimientos muestran un despoblamiento evidente.

\section{Caracterización de la población regional}

Un elemento clave, en términos de la constatación censal, es la sostenida tendencia al despoblamiento de las zonas altas y a la aparición de fenómenos demográficos que ponen en cuestión las posibilidades del desarrollo que puedan ser capaces de alcanzar los andinos. Así, se encuentra el debilitamiento de las cohortes de edad más capaces de alcanzar mejores niveles de productividad y el desequilibrio en la relación hombre-mujer, lo que se puede advertir en el siguiente cuadro estadístico.

La población rural pobre es notoriamente más elevada que la regional y, especialmente, que la urbana, expresándose con más fuerza en las comunas más apartadas de las urbes principales del sistema de asentamientos humanos de la región de Tarapacá. Lo relevante del análisis de la situación social es que no todos los indicadores son desfavorables en relación con la situación existente en la ciudad, sin embargo la falta de proyección estratégica, legitimada y deseable, de la vida andina, hace que la tendencia a la emigración hacia las zonas costeras y urbanas de la región siga aumentando (ver Cuadro 1).

Cuadro 1. Población regional Tarapacá 2012

\begin{tabular}{lrrrr}
\hline Región & \multicolumn{1}{c}{ Total } & Hombres & Mujeres & Masculinidad \\
\hline & 300.021 & 151.084 & 148.937 & 101.44 \\
Urbana & 287.287 & 142.982 & 144.305 & 90.08 \\
Rural & 12.734 & 8.102 & 4.632 & 174.91 \\
\hline
\end{tabular}

Fuente: Instituto Nacional de Estadísticas, Chile 2012.

\section{Minería y Espacio Social}

El trabajo minero, propio de la modernidad laboral en la región, ha tenido vínculos con la población local, los que han variado de acuerdo con las distintas inserciones y carácter del capital minero.

La minería metálica transnacional, emergente a partir de fines del siglo XX con la puesta en ejecución de tres grandes proyectos cupríferos de gran minería: Cerro Colorado, Quebrada Blanca y Doña Inés de Collahuasi, muestra características singulares respecto de ciclos extractivos que le antecedieron, como fueron los casos del salitre y la extracción de cobre de la década del 70.

En cuanto a la participación de la población local, a diferencia de los ciclos previos, la organización del trabajo y la estructura residencial de los campamentos de trabajadores no facilitan la incorporación de las familias, al menos en dependencias de la empresa, pues la localización de estas no propician la vida familiar, sino que está organizada solo para los trabajadores. El punto de conflicto principal es que el ethos laboral indígena incorpora estructuralmente al individuo y su familia, mientras que la estrategia moderna "desfamiliza" a los obreros.

Si bien la minería del cobre tiene incidencia en toda la región de Tarapacá, su impacto fundamental se vierte sobre su personal directo y comunidades locales más cercanas a los yacimientos, como es el caso de Huatacondo.

Los trabajadores mineros: el nuevo sistema de asentamientos humanos producido por la minería sigue basándose en la estructura de campamentos, donde se concentra espacialmente a los trabajadores en función de acercarlos a la faena productiva y mantener en su residencia una continuidad de los lazos de dependencia con la empresa.

Las características centrales son: un sistema de turnos de una semana de trabajo por una de descanso ( $7 \times 7)$, donde los trabajadores producen rasgos de enajenación debido al distanciamiento de la sociedad global en que permanecen durante un período importante de su vida. Asimismo, el buen trato en materia de alojamiento, alimentación y transporte los consigna como representantes de la "aristocracia obrera" chilena, que junto a contar con ingresos superiores al promedio les produce una distancia cultural respecto de los problemas de las mayorías. El otro rasgo central es que, al igual que en los campamentos antiguos, se les concentra en un espacio inmediatamente aledaño a la faena, pero con 
la diferencia de que durante la mitad de su tiempo (7x7) son "devueltos" a la sociedad nacional, en la que usufructúan de su red de servicios.

Estas características del sujeto minero producen condiciones de habitabilidad precaria en la región y condicionan notablemente su sociabilidad, identidad cultural, expresadas en su participación social. Esto se advierte en Cuadros 2 y 3 del presente artículo.

La presente información estadística es de realización propia y elaborada en el marco de la investigación de doctorado que realizó el autor en el 2008. Para recolectar los datos se censó a la población de las empresas Quebrada Blanca y Collahuasi que residen en condominios mineros - construidos en la ciudad de Iquique- en sus domicilios, debido a las dificultades para hacerlo en faenas y además por la relevancia científica de evitar sesgos de carácter laboral. Asimismo se trataba de encontrar a los sujetos en su residencia y no en su lugar de trabajo.

Se trabajó sin muestra, optándose por censar a la totalidad de las personas que se encontraban en sus hogares en el momento de la aplicación, que por las características de la rotación laboral por turno alcanzó al 50\% de la población.

\section{Migración y sociabilidad}

Este cuadro permite constatar que se trata de una emigración de carácter socioeconómico, donde otros factores son marginales (ver Cuadro 2). Este Cuadro, si bien en forma aislada no entrega mucha información, cuando se asocia claramente a otros aspectos demuestra que esta condición de la emigración incide en la relación con el medio local, particularmente con la cultura. A la vez, se muestra un rasgo que define el carácter de estas migraciones en Chile, no tienen opción y el mercado laboral no proporciona espacios para una búsqueda más selectiva. La obtención del puesto de trabajo por

Cuadro 2. Factores de emigración

\begin{tabular}{lcc}
\hline \multirow{2}{*}{$\begin{array}{l}\text { Razones de } \\
\text { emigración }\end{array}$} & \multicolumn{2}{c}{ Estadísticos } \\
\cline { 2 - 3 } & $\mathrm{N}^{\mathrm{o}}$ de personas & Porcentaje de personas \\
\hline Clima & 7 & 4,5 \\
Ingresos & 12 & 7,7 \\
Empleo & 131 & 84,5 \\
Otra & 5 & 3,2 \\
Total & 155 & 100,0 \\
\hline
\end{tabular}

Fuente: elaboración propia, 2008. sobre toda otra consideración influye sin contrapeso acerca de cualquier otro factor.

Desde el punto de vista de la sociabilidad y del capital social, esta población desplazada marca de manera muy profunda el carácter del espacio social minero, ya que tenderá a desarrollar una sociabilidad liviana y un capital social frágil, de muy difícil reposición frente al existente en su localidad de origen.

\section{Participación social y sociabilidad}

La población en general tiene una limitada participación en organizaciones comunitarias, lo que demuestra la falta de conexiones barriales y escaso capital social. La realidad de otros barrios de trabajadores en Iquique muestra una participación que supera el 50\% (ver Cuadro 3).

Esta información muestra además que esta participación se reduce al ámbito habitacional, es decir, al mantenimiento de las condiciones de habitabilidad existentes en el condominio, lo que refrenda la alta frecuencia de respuestas entregadas cuando se preguntaba acerca de la segunda prioridad en materia de aspecto más valorado de la residencia en Iquique, resaltando en primer lugar $-31 \%$ - la seguridad residencial existente en el condominio.

Potencialmente este constituye un considerable factor de interés común, que puede cristalizar en el futuro como factor de cohesión social. A la vez permite constatar que la relación con organizaciones donde resalta la identidad local no muestra un interés relevante, evidenciando un distanciamiento de los intereses y problemas de la localidad, los que en su caso particular ya estarían resueltos, llámense estos desempleo, delincuencia, basura y otros.

Este fenómeno de segmentación social, o bien segregación visto desde una perspectiva crítica, evidencia una ventaja apreciable respecto de otros sectores de trabajadores que residen en la localidad, pero asimismo constituye una amenaza desde el

Cuadro 3. Participación social en Iquique

\begin{tabular}{lcc}
\hline \multirow{2}{*}{ Participación } & \multicolumn{2}{c}{ Estadísticos } \\
\cline { 2 - 3 } & $\mathrm{N}^{\mathbf{o}}$ de personas & Porcentaje de personas \\
\hline Sí & 46 & 29,7 \\
No & 109 & 70,3 \\
Total & 155 & 100,0 \\
\hline
\end{tabular}

Fuente: elaboración propia. 2008. 
punto de vista del riesgo social en tanto sus bienes serían una atracción para aquellos grupos sociales excluidos del acceso a los mismos. Por ello es comprensible que su participación mayoritaria es la referida a los temas de seguridad del condominio.

La población aymara: la población originaria no es objeto central en los procesos de la nueva minería, ni como aporte laboral ni como productor de alimentos. Los modernos procesos mineros requieren obreros de alta calificación y los insumos alimentarios son trasladados desde grandes cadenas de producción.

El desarrollo minero ha tenido un gran impacto en la reconstrucción de los espacios sociales indígenas, si bien es cierto que no tiene la envergadura laboral de anteriores ciclos mineros, la población se ve afectada por la disputa del recurso agua. La actual situación de los asentamientos humanos aymaras, andinos y de valles, se inscribe en la fase de un cambio profundo en el marco territorial regional, porque los énfasis productivos determinan la consolidación de una perspectiva urbana en cuanto a la concentración y el disfrute de beneficios de las actividades productivas, independientemente de que estas se desarrollen en zonas rurales.

En cuanto a la minería, la alta calificación e individuación de ella no se hace compatible con las características educacionales de la población aymara, como tampoco su tendencia a la vida familiar que inhibe el desarrollo de una concepción obrera del trabajo. Asimismo, la pérdida creciente de los recursos hidráulicos, por concentrarse estos en las mineras, desahucia definitivamente las posibilidades de recuperación de las actividades agrícolas.

Un creciente problema derivado de la relación entre la minería y la población aymara tiene características ambientales, principalmente relacionados con la enajenación de los recursos hídricos, los que presentan un doble efecto perverso: la contaminación de las corrientes subterráneas y superficiales, como también la sobreexplotación de dichas fuentes.

El tema ambiental se encuentra condicionado por la existencia de una historia de relaciones asimétricas, donde se originan sus grandes desequilibrios ecológicos. Ejemplo de esto son la pérdida de su estrategia productiva basada en la agricultura y ganadería, como también las recientes expoliaciones de importantes fuentes de recursos hidráulicos para fines mineros.

En cuanto al sistema de asentamientos humanos, este tiene directa relación con la expoliación de sus recursos naturales de que están siendo objetos, principalmente por la minería, lo que incide en la pérdida definitiva de su estrategia productiva ancestral y limita completamente su relación con el mercado. Esto tiene como consecuencia final una disminución de su población y el quiebre de su patrón de asentamientos.

Las migraciones hacia la ciudad, la movilidad entre los distintos pisos ecológicos de la provincia, las propiedades rurales de familias que se han trasladado a la ciudad, la potencial producción agrícola y ganadera, son aspectos incidentes en la definición de situación de los aymaras, lo que determina sus prioridades y demandas.

En las décadas 80-90 la producción social del medio rural está marcada por transformaciones importantes, que involucran un cambio notable en la composición de la población, actividades y relaciones sociales. En esto ocupa un lugar de primerísima importancia la presencia y carácter del capital minero y la modernización de la sociedad chilena. Los cambios son de tal envergadura, que comprometen profunda y definitivamente las comunidades tradicionales, aumentando la emigración y amenazando definitivamente la residencia definitiva y estable en ellos, observándose una tendencia a que la permanencia en los asentamientos sea temporal e inestable.

La realización de un estudio realizado por la Universidad Arturo Prat entre 1991 y 2012 -financiado por la Cía. Minera Quebrada Blanca- que consistió en un Censo socioeconómico, flora y fauna del territorio aledaño al yacimiento que explota dicha empresa, permite contar con información fidedigna para dar cuenta de la evolución social de las poblaciones de Colonia Pintados, Huatacondo, Copaquire y Soga. Estos poblados ubicados en la pampa del Tamarugal y Precordillera representan en este artículo el impacto socioeconómico directo de la actividad minera en la realidad sociocultural.

La metodología de este estudio consistió en la realización de un censo socioeconómico semestral en los poblados considerados, mostrando la evolución de la población en cuanto a los aspectos familiares, educacionales, sanitarios, laborales y otros de relevancia socioeconómica y cultural.

Los últimos registros censales muestran, en la comparación de censos realizados durante veinte años en el territorio objeto de la investigación, la sostenida tendencia al despoblamiento de las zonas altas, el reemplazo de la población originaria y la 
aparición de otros fenómenos demográficos que ponen en cuestión sus posibilidades de desarrollo futuro. Esto deriva tanto del quiebre de la estructura productiva tradicional como de procesos ideológicos, determinando un cambio definitivo en la composición poblacional y en sus proyectos sociales colectivos.

Esto tiene como consecuencia final una disminución de su población, el quiebre de su patrón de asentamientos humanos y la transformación profunda de sus relaciones sociales. Un estudio realizado en el 2001 revela que la tendencia al despoblamiento, revertido entre 1991 y 1998 por los impactos socioeconómicos favorables al inicio de las actividades mineras, es un proceso consolidado y que el crecimiento poblacional alcanzado en dicho período a raíz de la dinámica económica impulsada por los proyectos mineros ha tenido una corta duración. Ello es demostrado por el hecho de que Copaquire ya no registra población permanente y Huatacondo revela un proceso de envejecimiento y desplazamientos cada vez más frecuentes hacia las localidades urbanas (ver Cuadro 4).

En su relación con la minería, la difusión de los beneficios se produce en los inicios de las actividades de instalación y montaje, donde la precariedad de los servicios propios los lleva a utilizar los recursos tradicionales existentes en las comunidades aledañas, especialmente en cuanto a empleo y servicios, pero esta participación es efímera y además en el largo plazo altera negativamente los procesos de emigración sostenida, acelerándolos.

La población del 2001 respecto de 1995 (punto más alto del crecimiento demográfico de la década del 90) corresponde a $47,9 \%$, demostrando la continuidad del despoblamiento alcanzado en este antiguo asentamiento humano, anulando el impacto positivo registrado hasta 1998 y moderando la caída significativa que se había registrado en el 2000.

Hacia el fin de la década del 2000 se produce un aumento de población caracterizado por reingreso de población que había emigrado de la localidad. La población alcanza en el último censo -2010 a 70 habitantes e indica cierta recuperación de población que había disminuido en esta década al compararla con la de los 90. Esta nueva población se trata principalmente de pensionados, en su mayoría originarios de Huatacondo.

Quizás uno de los fenómenos más cruciales que surgen de estas profundas transformaciones es la pérdida de autosustentabilidad por parte de las comunidades tradicionales, tanto en la producción como en la reproducción social, tanto en la satisfacción de necesidades económicas como en la construcción de redes sociales que permitan la autosuficiencia. Se trata, como diría Gorz, de una pérdida del sentido de comunidad, en tanto relaciones sociales basadas en una sociabilidad densa e íntima.

\section{La Reconstrucción del Espacio Social: la Nueva Sociabilidad}

Las transformaciones del espacio social, es decir, la intervención del territorio físico por las adecuaciones materiales y culturales del ser humano, se refieren a sus efectos en la región de Tarapacá y se relacionan con los cambios estructurales derivados de los proyectos de producción minera. Estos dan lugar a nuevas prácticas sociales que a su vez concluyen en resignificaciones de la vida cotidiana, que entre las más importantes connotaciones que

Cuadro 4. Distribución de la población por sexo y localidades, años 2001 al 2010

\begin{tabular}{|c|c|c|c|c|c|c|c|c|c|c|c|c|c|c|c|c|}
\hline \multirow{2}{*}{ Localidad } & \multicolumn{4}{|c|}{ Año 1991} & \multicolumn{4}{|c|}{ Año 1995} & \multicolumn{4}{|c|}{ Año 2000} & \multicolumn{4}{|c|}{ Año 2001} \\
\hline & $\mathrm{H}$ & M & AS & $\%$ & $\mathrm{H}$ & M & AS & $\%$ & $\mathrm{H}$ & $\mathrm{M}$ & AS & $\%$ & $\mathrm{H}$ & M & AS & $\%$ \\
\hline Copaquire & 8 & 4 & 12 & 12,5 & 1 & 1 & 2 & 2 & 0 & 0 & 0 & 0 & 0 & 0 & 0 & 0 \\
\hline Huatacondo & 47 & 37 & 84 & 87,5 & 53 & 43 & 96 & 98 & 20 & 12 & 32 & 100 & 29 & 17 & 46 & 100 \\
\hline Total & 55 & 41 & 96 & 100 & 54 & 44 & 98 & 100 & 20 & 12 & 32 & 100 & 29 & 17 & 46 & 100 \\
\hline \multirow{2}{*}{ Localidad } & \multicolumn{4}{|c|}{ Año 2007} & \multicolumn{4}{|c|}{ Año 2008} & \multicolumn{4}{|c|}{ Año 2009} & \multicolumn{4}{|c|}{ Año 2010} \\
\hline & $\mathrm{H}$ & M & AS & $\%$ & $\mathrm{H}$ & M & AS & $\%$ & $\mathrm{H}$ & M & AS & $\%$ & $\mathrm{H}$ & M & AS & $\%$ \\
\hline Copaquire & 0 & 0 & 0 & 0 & 0 & 0 & 0 & 0 & 0 & 0 & 0 & 0 & 0 & 0 & 0 & 0 \\
\hline Huatacondo & 32 & 19 & 51 & 100 & 26 & 31 & 57 & 100 & 29 & 33 & 62 & 100 & 100 & 35 & 35 & 100 \\
\hline Total & 32 & 19 & 51 & 100 & 26 & 31 & 57 & 100 & 29 & 33 & 62 & 100 & 100 & 35 & 35 & 100 \\
\hline
\end{tabular}

Fuente: elaboración propia. 2010. 
traen consigo presentan un nuevo cuadro de cohesión social e identidad cultural, que en suma expresan una nueva sociabilidad.

Las nuevas estructuras y dinámicas socioculturales se producen tanto en el ámbito urbano como en el rural, pero en un marco social donde estas realidades, otrora lejanas, aparecen cercanas y continuas a raíz de dinámicas económicas sostenidas por nuevos medios de comunicación, especialmente de transporte e información.

Estas dinámicas económicas, con resultados socioculturales importantes, ocurren en un escenario de globalización, el que impulsa aún más la reconfiguración espacial, haciendo que antiguas relaciones, costumbres y nociones existentes en el orden social tradicional comiencen a cambiar drásticamente.

En este sentido, las nociones de tiempo-espacio, urbano-rural, tradicional-moderno han cambiado debido a las nuevas formas de convivencia humana, impulsada por la existencia de nuevas tecnologías, especialmente de transportes e información, hacen que estas categorías pares comiencen a perder sentido.

En la actualidad las relaciones se construyen sobre la base de una realidad más veloz y cercana, aunque muchas veces esto sea a expensas de que las interacciones se constituyan sobre una base virtual. La dicotomía rural-urbano comienza a perder validez, pues la revolución del transporte y de las comunicaciones, expresadas en la televisión, la telefonía, la informática y el acceso a vehículos motorizados permite que dicho par se transforme en un continuo de permanente movimiento. Por su parte lo tradicional-moderno se va inclinando hacia lo segundo, por las facilidades de acceso a la información y a la impronta de la vida actual.

Puede decirse con cierta seguridad que el espacio donde se producen las interacciones ha cambiado con notoriedad, desde una idea de lugares definidos, que señalaban con evidencia las distancias, a una idea distinta de flujos, donde el espacio está en permanente y rápida recomposición, claramente móvil.

Las transformaciones sociales suscitadas en los últimos años, debido a la nueva modernización causada por la nueva revolución tecnológica, han obligado a las ciencias sociales a modificar sus perspectivas tradicionales.

Las coordenadas de espacio y tiempo, que permiten a los seres humanos situarse en su realidad, también han sufrido los efectos de esta transformación de dimensión planetaria, llevándolos a reinterpretar su noción de modernidad. Asimismo, esta dinámica conduce a una nueva dialéctica de las relaciones sociales y espaciales, construyendo una nueva sociabilidad.

Sin embargo no debe entenderse la relación espacio-sociedad en una sola dirección, pues se influyen mutuamente, en su estructuración y sus movimientos, dando lugar a nuevas formas y contenidos. En este marco se puede decir que la sociabilidad, como expresión de lejanías o cercanías sociales, depende tanto del espacio como de aspectos inmateriales como la ideología.

En esta reconfiguración del espacio es importante recordar lo planteado por Georg Simmel hace más de un siglo respecto de la relación entre sujetos y objetos, los que tienen cada una circulación más rápida, con sujetos comprometidos en una circulación más veloz. Ciertamente, hoy en el siglo XXI, tal observación de este autor clásico de la sociología se ve reafirmada de manera contundente.

Haciéndose cargo de tal aseveración simmeliana, los autores británicos Lash y Urry contraponen las interacciones de las sociedades tradicionales y las modernas, siendo por oposición las segundas de carácter pasajeras, intensas y diversas, de corta duración. Estas tendrían plena validez en los procesos de transformación del espacio social que ocurren hoy en las provincias de Iquique y del Tamarugal a propósito de la dinámica del capital transnacional y de la incorporación de nuevas tecnologías en este medio.

A fin de entender los nuevos escenarios donde la relación capital-trabajo se establece, produciendo formas y modalidades de interacción con la sociedad, debe entenderse que el orden capitalista actual está signado por la globalización, cuyos alcances trascienden la dimensión económica y alcanzan la totalidad de las dimensiones de la vida humana. Con certeza, los efectos de la revolución tecnológica, las nuevas relaciones económicas y las reestructuraciones del Estado, alcanzan al conjunto de la sociedad humana, pero a la vez producen fenómenos particulares debido a las características específicas de las colectividades sociales.

Las elaboraciones de Georg Simmel, hace aproximadamente un siglo, marcan un punto de modernización anterior, equivalente al punto de inflexión actual en tanto la modernización sostenida en la revolución tecnológica desatada hace treinta años comienza a penetrar la sociedad al punto de transformar las pautas y configuraciones sociales. 
En la actualidad la relación entre el espacio y la sociedad tiene mayores evidencias que en los tiempos que hacía sus observaciones el autor, pero como se observa ya existía un conocimiento expresado seguramente por reflexiones y discusiones relativas al tema. No cabe duda de que esas discusiones tempranas fueron los primeros planteamientos frente a una realidad insoslayable y evidente como es hoy la construcción social del espacio.

La circulación del capital transnacional, minero en la realidad específica del estudio, es determinante de cambios importantes en el sistema de asentamientos humanos producidos por el carácter de sus flujos, incidiendo en la alteración de espacios sociales anteriores.

Una valiosa afirmación del importante sociólogo catalán Manuel Castells, que puede relacionarse con las características de los procesos socioeconómicos y culturales que acaecen en el norte chileno, es que este se encuentra conectado a las dinámicas de la globalización en tanto se localiza en una de las cuatro posiciones dominantes de la actual división espacial del trabajo: la de elaboradores de productos sin transformar, basados en recursos naturales. Este nuevo modelo territorial solo articula e integra aquellos territorios que necesita por ser funcionales y rentables en la acumulación capitalista.

La producción y reproducción capitalista será determinante en la estructuración del espacio social, pues tenderá a conformar los espacios habitados y relacionados directamente con la circulación del capital minero de acuerdo con sus características y necesidades. Tenderá a producir beneficios efímeros y concentrados en escasos individuos y territorios.

Uno de los grandes temas sostenidos por Manuel Castells, a propósito del carácter actual de la sociedad, se refiere a la identidad cultural en tanto estaría sometida a la crisis y constante reestructuración que, a la vez, impactaría el horizonte significacional de los sujetos. En el caso particular de la presente investigación sus transformaciones estarían conduciendo a una crisis o al establecimiento de nuevas identidades en la provincia de Iquique. Lo nuevo sería la sociedad regional cada vez más estamentaria en lo interno y por otra parte su conexión al exterior por los influjos de la globalización.

Estos rasgos planteados no se representan de manera nítida en todos los estamentos sociales, realidad que caracteriza a América Latina, pero sí lo hace en aquellas realidades más cercanas a los circuitos de valorización del capital transnacional, como es el caso de los espacios rurales y urbanos ligados a la minería transnacional de la provincia de Iquique. A la vez, el impacto no se refleja solo en el carácter material y en la esfera del poder, sino que alcanza los aspectos subjetivos que orientan el comportamiento en la vida cotidiana de los sujetos. Esto se convierte en las nuevas bases para la construcción de identidades.

Los enfoques provenientes de la geografía social moderna destacan por su énfasis en la dinámica del espacio, transformándose estos en una variable socialmente construida, constituidos más allá de características físicas, trascendiendo así la conceptualización tradicional que enfatizaba el carácter receptor, soporte y localización. Esto se relaciona con las ideas de flujos por sobre las de lugar que son planteadas en la investigación. Asimismo se incluyen las ideas de redes y nodos que explican territorialmente las dinámicas propias del orden globalizado actual. En este sentido, los autores Saskia Sassen y Milton Santos son las perspectivas geográficas que se incluyen en la investigación, debido a su relevancia en la nueva geografía del desarrollo.

Milton Santos hace mención a un tema clave que produce diferenciación en la sociedad, esto es la tensión creciente que se establece entre lo global y lo local, los que tienen lógicas diferentes, el local vivido por todos los vecinos y el global regido por un proceso racionalizador y un contenido ideológico de origen distante. El primero estaría al servicio de todos y el segundo al servicio de algunos. Asimismo, de este mismo autor, sus trabajos acerca del sentido del tiempo y el espacio, en tanto construcción, es cultural, son determinantes en la conformación de cierta concepción del mundo y a la vez precondiciones de la racionalidad en ciertos espacios.

Finalmente, deben considerarse algunas ideas expuestas en la publicación Sociología de la movilidad espacial del sociólogo hispano Eduardo Bericat Alastuey, quien relaciona la mayor capacidad de movilización que en la actualidad tienen los sujetos y el carácter de su relación con la sociedad. Al respecto recurre a Georg Simmel y Emile Durkheim, a fin de retomar sus ideas del espacio y la movilidad, respectivamente, que hoy son materias de vital importancia para entender la compleja relación señalada.

La transformación del espacio social y con ello de la sociabilidad en los espacios urbanos y rurales, está asociada a que las relaciones sociales son más 
diversificadas y en todo caso tienen que ver con una ruptura de las pautas de dichos encuentros. En este sentido los movimientos entre distintos espacios y también los producidos al interior de los mismos, son factores importantes en dichas fisuras de comportamiento.

Las migraciones permanentes y definitivas, que han cambiado en su frecuencia y razón, constituyen entonces un factor de primera línea frente a la transformación del espacio social y el carácter de la sociabilidad producida. El mismo autor se refiere a fenómenos asociados a la dinámica social, como el tiempo de estancia en cada uno de los destinos a que se accede, también la cantidad de movimientos del conjunto de viajes y la homogeneidad o heterogeneidad de la longitud de los viajes. También la recurrencia de los destinos, porque una repetición reiterada no es igual a uno esporádico.

\section{Transformación del espacio rural y la ciudad}

Como se ha planteado, la dicotomía urbano-rural hoy se expresa como un continuo, lo distante como cercano y lo distinto como semejante. Son cada vez espacios mutuamente inclusivos. El concepto de flujo ha disminuido la importancia que tenía la categoría lugar, pues este tiende a ser menos duradero, incesante y rápidamente reconstruido. La televisión, la informática y las nuevas tecnologías de transporte permiten que los sujetos puedan transitar con más frecuencia y más propiedad por espacios que antes tenían una mayor autonomía y distancia entre sí.

Desde un punto de vista general, dadas las nuevas condiciones de la producción y la reproducción social, la realidad rural está sometida a intensas discusiones, tanto en materia práctica como teórica. En ese sentido actualmente tanto el concepto de rural como el objeto de estudio de la sociología rural están sometidas a constantes discusiones, lo que se explica en tanto el carácter más estrecho que tiene con lo urbano, como también con la constante y rápida transformación a que se ve expuesta.

Por otra parte las transformaciones sociales, en el marco del actual proceso de modernizaciones, ha generado profundas tensiones en las significaciones rurales, debido a la celeridad del cambio y la permanente tensión de los procesos de construcción social, así se entiende el siguiente planteamiento frente a tradicionales áreas de competencia y construcción de objetos de disciplinas como la geografía, que tendían a apoyarse en la naturaleza física de su objeto.

Por cierto y en la misma lógica que se ha estado planteando esta transformación de los ejes urbanorural, debe considerarse de manera importante el impacto de la globalización, como fenómeno que señala esta tendencia de la sociedad actual a hiperdesarrollar su capacidad de conexión, al interior de las realidades locales y en su contacto permanente con las realidades exteriores. En este sentido la globalización otorga un carácter epocal a las circunstancias actualmente vividas. Pero aún hay más, no se trata de un fenómeno puramente económico, pues su carácter es esencialmente cultural. Tampoco trae solo beneficios, sino también exclusiones.

En este sentido, el sociólogo polaco Zygmunt Bauman señala la profundidad y la extensión de estos impactos y que generan una dinámica no vista antes entre lo global y lo local. Esto tiene neta incidencia sobre la condición humana, ya que se relacionan con aspectos básicos de la existencia, como las categorías espacio-tiempo.

Es cierto que las posibilidades de movilizarse en la actualidad son extraordinariamente abundantes, pero esto no alcanza a toda la población, generando nuevas instancias de fijación al espacio y agregando nuevas fuentes de estratificación social. Siguen existiendo distintos mundos en la globalización, que producen diferentes prácticas sociales y, por ende, conflictos sociales entre opuestos.

\section{Conclusiones}

La más reciente presencia del capital minero en la región de Tarapacá se origina en la década del 80, a propósito de la atracción que suscita en las corporaciones transnacionales la nueva ley minera, muy proclive a los intereses del capital. Las modificaciones de dicha ley impulsan a la minería transnacional, emergente a partir de la década del 90 con la puesta en ejecución de tres grandes proyectos cupríferos de gran minería; Cerro Colorado, Quebrada Blanca y Doña Inés de Collahuasi.

Sus características, junto al hecho de constituir un mayor peso en el Producto Geográfico Bruto de la región, incidiendo de manera más determinante en la economía regional, no constituyó un aumento notable de Fuerza de Trabajo por Unidad productiva, a causa de sus características intensivas en capital. Así, las tres unidades productivas no sobrepasan 
en promedio 500 trabajadores cada una, pero su producción es significativamente mayor que las del período pasado.

El peso de estas inversiones y su efecto sobre la economía nacional hace que su desarrollo tenga influencia dominante sobre el escenario regional, transformando el espacio social más allá de su actividad productiva. Esto ocurre tanto en el espacio rural, en las comunidades más cercanas a los yacimientos, como en los espacios urbanos, particularmente en su capital regional.

En cuanto a la participación de la población local, a diferencia de los casos anteriores la organización del trabajo y la estructura residencial de los campamentos de trabajadores no facilitan la incorporación de las familias, al menos en dependencias de la empresa, pues la localización de estas no propicia la vida familiar, sino que está organizado solo para los trabajadores. El punto de conflicto principal es que el ethos laboral indígena incorpora estructuralmente al individuo y su familia, mientras que la estrategia moderna "desfamiliza" a los obreros.

A pesar de que el nuevo sistema de asentamientos humanos producido por la minería sigue basándose en la estructura de campamentos, donde se concentra espacialmente a los trabajadores en función de acercarlos a la faena productiva y mantener en su residencia una continuidad de los lazos de dependencia con la empresa, las condiciones tecnológicas actuales cambian el tipo de relación establecida con el entorno y con los grupos familiares.

Sin embargo, lo nuevo es que el modelo minero actual incluye la construcción de conjuntos residenciales para los trabajadores y sus familiares, en conglomerados urbanos conocidos como campamentos mineros. Aquí se construyen relaciones sociales con significado propio, a diferencia de otros -antiguos y nuevos- grupos sociales existentes en la ciudad.

En los condominios mineros construidos en la ciudad de Iquique es donde más se evidencia el carácter singular de las relaciones sociales contraídas en el entorno minero, porque sus vínculos con los temas de la ciudad tienden a ser superficiales, careciendo de identidad con los intereses de la ciudad a la que territorialmente pertenecen. Asimismo, las relaciones internas, entre los residentes mineros del condominio, tienden a ser impersonales y fugaces. Los mayores recursos financieros que tienen las familias y en consecuencia el mayor acceso a los bienes materiales, especialmente equipos de alta tecnología, les proporcionan capacidad para resolver individualmente sus problemas y necesidades, generando condiciones para construir un espacio social caracterizado por el individualismo y la escasa identificación con el condominio y la ciudad. Hay, en consecuencia, un mayor acceso al capital económico pero a costa de un débil capital social.

En tanto se han desarrollado los proyectos mineros y madurado sus relaciones de producción, la contradicción entre lo económico v/s lo sociocultural se ha ido evidenciando. La disminución de capital social y una sociabilidad de menor densidad son factores que llaman a observar críticamente el desenlace futuro de la relación economía y sociedad en este escenario de sólida presencia de capital minero transnacional.

\section{Referencias Citadas}

\footnotetext{
Bauman, Zigmunt

1996 La Globalización: consecuencias humanas. Fondo de Cultura Económica, México.

Bericat, Eduardo

1994 Sociología de la Movilidad Espacial, Ed. Siglo XXI, España.

Castells, Manuel

1997 La era de la Información. Ed. Alianza, Madrid.

Entrena Durán, Francisco

1998 Cambios en la Construcción Social de lo Rural,

Ed. Tecnos, España.

Lash S. y Urry J.

1998 Economía de signos y espacios. Amorrortu Editores. B. Aires.
}

Simmel, Georg

1977 Estudio sobre las formas de socialización. Ed. Alianza, Madrid.

Santos, Milton

1996 De la Totalidad al lugar. Ed. oikos - tau. España.

Santos, Milton.

1996 Metamorfosis del Espacio Habitado. Ed. Oikos - tau, España.

Universidad Arturo Prat

1991 a 2010 Informes de investigación "Monitoreo ambiental en el área de explotación Minera Quebrada Blanca". 


ANDROS IMPRESORES

www.androsimpresores.cl 
